\title{
Review Article \\ Protective Effects of Polyphenols Present in Mediterranean Diet on Endothelial Dysfunction
}

\author{
K. Stromsnes $(\mathbb{D}$, C. Mas-Bargues $\mathbb{D}$, J. Gambini $\mathbb{D}$, and L. Gimeno-Mallench \\ Freshage Research Group, Department of Physiology, Faculty of Medicine, University of Valencia, CIBERFES, INCLIVA, \\ Avenida Blasco Ibañez, 1546010 Valencia, Spain \\ Correspondence should be addressed to J. Gambini; juan.gambini@uv.es
}

Received 30 April 2020; Revised 2 July 2020; Accepted 18 July 2020; Published 6 August 2020

Academic Editor: Maria U. Moreno

Copyright (C) 2020 K. Stromsnes et al. This is an open access article distributed under the Creative Commons Attribution License, which permits unrestricted use, distribution, and reproduction in any medium, provided the original work is properly cited.

\begin{abstract}
Endothelial dysfunction tends to be the initial indicator in proinflammatory state and macro- and microvascular complications, such as atherosclerosis and cardiovascular diseases. It has been shown that certain compounds in diet can generate beneficial effects on cardiovascular disease due to its interactions with endothelial cells. Thus, this review is aimed at investigating whether certain polyphenols present in the Mediterranean diet, specifically catechin, quercetin, resveratrol, and urolithin, could exert positive effects on endothelial dysfunction. After analysis of numerous papers, we found that polyphenols aiding endothelial function is beneficial not only for patients with cardiovascular disease, diabetes, or endothelial dysfunction but for all people as it can improve the effects of aging on the endothelia. The additional benefit of these polyphenols on weight loss further improves health and lowers the risk of several diseases, including those caused by endothelial dysfunction. However, it is important to note that the dosages in the majorities of the studies mentioned in this review were of supplemental rather than nutritionally relevant quantities, and therefore, the recommended dosages are difficult to determine.
\end{abstract}

\section{Introduction}

1.1. Endothelial Function. Vascular endothelial cells line the entire circulatory system, from the heart to the smallest capillaries, and play a crucial role in maintaining cardiovascular homeostasis, attenuating vascular inflammation and controlling blood flow and vascular tone $[1,2]$.

A shift in the functions of the endothelium can cause dysfunction through vasoconstriction, proinflammatory and prothrombic states $[2,3]$. Endothelial dysfunction tends to be the initial state in macrovascular complications such as coronary artery disease, peripheral arterial disease, and stroke. It may also lead to microvascular complications such as nephropathy, neuropathy, and retinopathy. Numerous strategies have been developed to protect endothelial cells, of which the role of polyphenolic compounds in modulating the differentially regulated pathways has been proven to be beneficial [4]. Control of the endothelial functions is achieved through the production and release of various mediators, such as the vasoconstrictor endothelin-1 (ET-1) and relaxant nitric oxide $(\mathrm{NO})$ that modify the responsiveness of the underlying vascular smooth muscle $[3,5]$ (see Figure 1) [6].

The figure shows the different local factors that directly affect the function of endothelial cells. Vasodilators and vasoconstrictor modify the caliber of blood vessels. Coagulating and anticoagulating factors permit an optimal control of the hemostasis and impede spontaneous coagulations, which are also influenced by inflammatory mediators. Together with angiogenic factors and proliferation regulation, optimal cell replacement and reparation are achieved. Generally, antiinflammatory mediators and dilating, proliferation, angiogenic, and procoagulant factors tend to be beneficial for diseases related to the cardiovascular system. However, these should exist in a balanced state with their opposing factor/mediator to ensure correct endothelial function.

1.2. Polyphenols. Polyphenols are chemical compounds synthesized by plants containing at least one aromatic ring and a hydroxyl group [7]. They are the main source of 


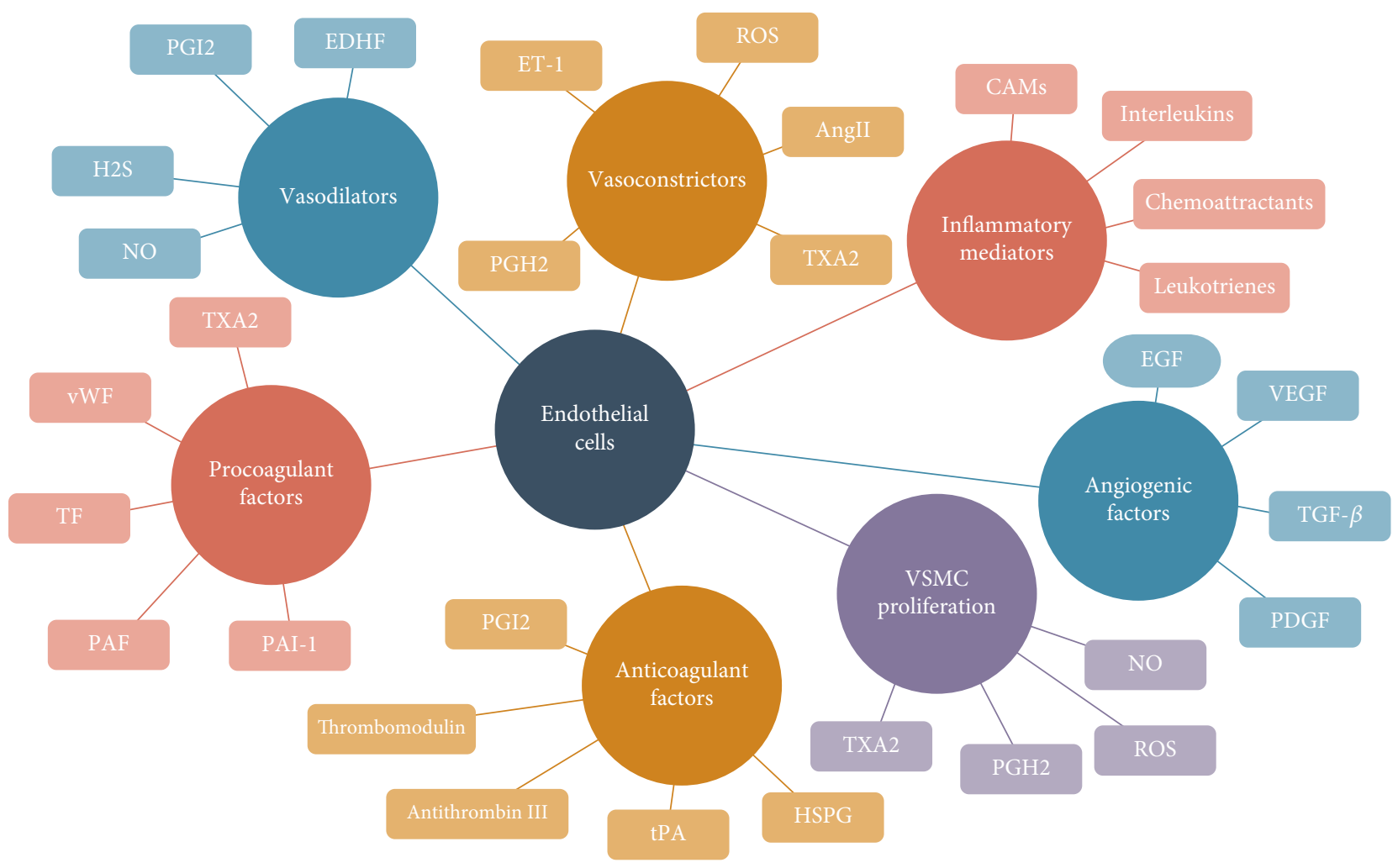

Figure 1: Endothelial function mediators [6], modified from Sena 2018.

antioxidants in diet, as they are abundant in plants, including fruits, nuts, vegetables, and cereals, as well as derived beverages such as tea, coffee, and wine [8]. There are more than 8000 known polyphenols, which are classified into phenolic acids, stilbenes, phenolic alcohols, lignans, and flavonoids $[8,9]$. Polyphenols have spiked great interest in the scientific community due to their health benefits, particularly in relation to cardiovascular diseases [10]. These exerted effects are suggested to include anti-inflammatory, antithrombotic, anticoagulant, antimicrobial, vasodilatory, and anticarcinogenic protection [10].

1.3. Mediterranean Diet. The Mediterranean diet is widely known for being rich in fruits, vegetables, legumes, nuts, and grains with a moderate intake of red wine and reduced consumptions of meat and dairy. Due to the antioxidant and anti-inflammatory properties of plant foods and the low intake of saturated fats, those following a Mediterranean diet have been shown to have lower incidence of obesity, insulin resistance, endothelial dysfunction, and cardiovascular disease $[11,12]$.

Red wine is one of the components present in this diet and is thought to be responsible for the "French-paradox", which describes the low risk of cardiovascular disease despite a diet rich in saturated fats and cholesterol [13]. Red wine contains, among other compounds, quercetin, catechin, resveratrol, and ellagic acid; the latter of which urolithin is obtained. Other fruits such as cherries, strawberries, grapes, and apples also contain catechin, quercetin, and ellagic acid.
Additionally, vegetables such as onions, cabbage, cauliflower, and capers contain important levels of quercetin. Walnuts and peanuts present both resveratrol and ellagic acid. All these compounds make up important components of the Mediterranean diet, and therefore, in this paper, we review the influence of these four polyphenols on overall health, mainly focusing on their effects on endothelial function and cardiovascular health.

1.4. Aging. Out of the theories of aging, those related to endothelial dysfunction include the theory of free radicals, mitochondrial dysfunction, the role of the insulin pathway, telomeric attrition, and senescence [14].

The idea of cellular senescence contributing to the aging process is supported by the finding that senescent cells halt normal function, irreversibly cease dividing, and accumulate in aging organisms, thereby causing dysfunction through inflammation and atherosclerotic plaques [15]. Endothelial cell senescence is induced by multiple factors, such as oxidative stress, vascular inflammation, and dysregulation of the cell cycle, which causes structural and functional changes, such as increased permeability of the endothelium, alterations in mitochondrial biogenesis, impairment of vascular repair, and angiogenesis [16]. Another hallmark of aging is telomere attrition. In human aortic endothelial cells, the inhibition of telomere function induces the expression of ICAM-1 and reduces eNOS expression, indicating that endothelial function is impaired in aging and cellular senescence $[14,17]$. The four polyphenols outlined in this review have 
been shown to have mediating effects on both ICAM-1 and eNOS expressions, indicating that they might have the ability to slow the shortening of telomeres caused by aging.

The IIS pathway is the most conserved aging-controlling pathway in evolution, and among its multiple targets are the FOXO family of transcription factors and the mTOR complexes. The two nutrient sensors, AMPK and sirtuins, act in the opposite direction to IIS and mTOR, and their upregulation favor healthy aging [14]. Mitochondrial function becomes perturbed by aging-associated mtDNA mutations, and defective mitochondrial function generates ROS that, below a certain threshold, induce survival signals to restore cellular homeostasis, but at higher or continued levels contribute to aging [14]. There is compelling evidence that polyphenols such as catechin, quercetin, and resveratrol inhibit mitochondrial ATP and thereby increase AMP levels and activation of AMPK, which promotes mitochondrial biogenesis and healthy aging [18]. Furthermore, resveratrol protects from metabolic damage and improve mitochondrial respiration in a PGC- $1 \alpha$-dependent manner [19].

As we will see, several polyphenols improve endothelial function by acting on mediators that are also described in the hallmarks of aging, such as telomerase attrition and mitochondrial function.

1.5. Metabolism. Recent studies have shown cell communication pathways linked to diabetes mellitus and obesity and how they may affect inflammatory and vascular health [20]. Therefore, a model to monitor endothelial dysfunction is the induction of diabetes, which is widely associated with cardiovascular diseases. Another measurable factor is obesity, which increases the risk of insulin resistance, disrupts vascular homeostasis, and contributes to endothelial vasodilator dysfunction and subsequent hypertension [21]. There is compelling evidence that the polyphenols outlined in this review can aid in weight loss and thereby decrease the risk of vascular metabolic diseases. Thus, consumption of polyphenols to maintain a healthy weight could decrease the risk of, and potentially even prevent, endothelial dysfunction before symptoms arise. Moreover, evidence shows that resveratrol increases lipolysis not only in obese animals but also in healthy, aged mice [22].

Based on this evidence, we propose in this review to study the role of quercetin, catechin, resveratrol, and urolithin on the endothelial dysfunction and related cardiovascular diseases.

\section{Quercetin}

Quercetin is a polyphenol belonging to the flavonoid family with anti-inflammatory, antioxidant and possible senolytic properties, among others [23-25]. It can be found in many plant foods, such as onions, apples, and blueberries, as well as in tea and red wine. Quercetin is not only beneficial in diabetic or inflammatory conditions; it also induces reduction of fatty acid and triacylglycerol synthesis and inhibits LDL oxidation in hepatocytes of healthy rats, thereby promoting cardiovascular protective functions [26, 27].

In regard to the mechanisms of the control of endothelial function, Akt, a downstream protein effector of PI3K, plays an important role in improving vasodilatory actions of insulin in human endothelium, thereby providing protective effects [28]. In this context, quercetin increases Akt phosphorylation and subsequently eNOS phosphorylation and NO production in response to insulin through inflammatory pathways in diabetic rats [29]. Additionally, quercetin at concentrations of $50 \mathrm{mg} / \mathrm{kg}$ exerts vasoprotective effects against endothelial dysfunction induced by endoplasmic reticulum stress. These effects are mediated by increased expression of VEGF in streptozotozine-induced diabetic rats [30], which is a model to study endothelial function.

ICAM-1 plays an essential role in inflammatory responses. The TNF- $\alpha$-induced expression of ICAM- 1 was shown to be reduced in an in vitro study with human endothelial cells when treated with $10-50 \mu \mathrm{M}$ quercetin [31]. Another study showed a significant inhibitory effect of $50 \mu \mathrm{M}$ treatment of quercetin on VCAM-1, ICAM-1, and E-selectin expressions. Inhibitory effects on COX-2 and iNOS protein levels were also found when treated with concentrations of 5-50 $\mu \mathrm{M}$ [32]. Quercetin has also been shown to activate the expression of the protein HIF-1 $\alpha$, a key transcription factor in the induction of the VEGF gene, as well as in regulating glucose and hypoxia homeostasis, potentially through its antioxidative properties, when treated with concentrations of $10 \mathrm{mM}$ [33].

Furthermore, quercetin has been shown to reduce body weight gain in high-fat diet-fed mice by lowering serum lipid levels and hepatic lipid accumulation. This is mediated through the downregulation of cytochrome p450 2b genes, which intervene in the synthesis of steroids, such as cholesterol, and are therefore important in hepatic lipid homeostasis $[34,35]$. However, in this study, quercetin was administered in $0.3 \% \mathrm{w} / \mathrm{w}$ dosages to rats in order to examine quercetin's effect in hepatic lipid metabolism [34]. This dosage indicates that a $70 \mathrm{~kg}$ individual would have to consume approximately 200 grams of quercetin supplements to achieve the same results.

Even though some studies have concluded that quercetin might not be effective as a senolytic in endothelial cells, a clinical trial conducted in 2019 showed that quercetin is effective in decreasing senescent cells $[25,36]$. However, as this is the first direct evidence that senolytics are effective in humans, further research is necessary to confirm the effects of quercetin in regard to senescence.

These results show that quercetin exerts multiple healthpromoting effects through an increase in vascular endothelial mediators, as well as possible weight loss, anti-aging, and senolytic benefits.

\section{Catechin}

Catechin is a flavanol present in many plants and is especially abundant in berries, cacao, and green tea [37]. A positive correlation between green tea and cardiovascular health has been found due to catechin's antioxidant, anti-inflammatory, antihypertensive, and antihyperlipidemic properties [38].

Catechin supplementation has been shown to significantly increase flow-mediated dilation and significantly reduce pulse wave velocity, diastolic blood pressure, and augmentation index, which are parameters for cardiovascular health in humans. This has been shown in several studies 
performed in both young and elderly healthy individuals as well as in individuals with cardiovascular disease risk through the administration of pure catechin supplements in quantities between 20 and $300 \mathrm{mg} /$ day and extracts from foods rich in catechins, such as apples, grapes, and cacao in quantities estimated between 7.5 and $146 \mathrm{mg} /$ day in a time period ranging between 2 and 12 weeks $[39,40]$.

Furthermore, one study showed that even in exceptionally low quantities, catechin exerted protective effects. The intake of red grape polyphenol extract containing only $2.72 \mathrm{mg}$ catechin was shown to improve endothelial function in patients with cardiovascular disease. In this study, 30 male patients with coronary heart disease were randomly assigned into two groups where one received polyphenol extract dissolved in water, and a placebo group received water without supplementation. The group consuming polyphenol extract showed significant increased flow-mediated dilatation compared to the placebo group, with a peak at $60 \mathrm{~min}$ after intake [41].

In addition to the cardiovascular and endothelial protective effects of catechin, it has also been shown to reduce body fat. Interventional studies of 3 daily cups of green tea after meals showed an average metabolic boost of about one hundred calories a day by promoting lipidic oxidation [42]. A possible explanation for why green tea consumption prolongs diet-induced thermogenesis is catechin's ability to inhibit catechol-O-methyltransferase, the enzyme that degrades norepinephrine [43]. However, in other studies, catechol-O-methyltransferase was not shown to be suppressed after consumption of catechin [44]. Therefore, a more plausible explanation for its thermogenesis-promoting effects is through brown adipose tissue signaling through evoking white adipocyte beiging and suppression of adipogenesis [45].

Moreover, daily intake of catechin-comprised red wine extract has shown to have a beneficial effect on aortic expression of endothelial dysfunction biomarkers. These include VCAM-1, ICAM-1, E-selectin, and LOX-1 as well as proinflammatory cytokines such as TNF- $\alpha$ and IL- 6 in hyperhomocysteinemic mice [46]. Catechin also improves redox imbalance and mitochondrial dysfunction, a prominent feature of cardiovascular disease, by stimulating phosphorylation of AMPK and ACC, inhibiting the key enzymes of de novo lipogenesis, and blocking the TNF- $\alpha$ induced insulin signaling pathway $[45,47]$. Furthermore, it significantly induces PGC- $1 \alpha$, which in turn stimulates mitochondrial proliferation [48].

These results suggest that consumption of catechin-rich food could be useful in combatting endothelial dysfunction both through direct signaling pathways, as well as indirectly through reducing risk factors of obesity-related metabolic diseases, such as diabetes and cardiovascular disease.

\section{Resveratrol}

Resveratrol is one of the most studied plant secondary metabolites [49]. Experimental studies have shown benefits of resveratrol on the cardiovascular system through blockage of low-density lipoprotein, cholesterol peroxidation, reduction of ROS, and prevention of platelet aggregation by promoting vasodilation through $\mathrm{NO}$ synthesis and inhibition of ET-1 [50-53].

In a randomized, double-blind, placebo-controlled study on 24 hypertensive patients with endothelial dysfunction, a single dose of $300 \mathrm{mg}$ of resveratrol supplement was shown to increase branchial flow-mediated dilation response and thereby improve endothelial function [54]. In a similar study, resveratrol improved flow-mediated dilation of the brachial artery of 19 overweight men and women with hypertension in a dose-dependent manner, with an increase from $4.1 \pm 0.8 \%$ measured in the placebo group to $7.7 \pm 1.5 \%$ after the intake of $270 \mathrm{mg}$ resveratrol 3 times weekly [55].

Studies in vitro further confirm the beneficial effects of resveratrol on endothelial metabolism and inflammation. In a study with TNF- $\alpha$-activated endothelial cells, the polyphenol intervention of $5-20 \mu \mathrm{M}$ significantly reduced the levels of VEGF, ROS, and proinflammatory mediator such as IL-8 and ICAM-1 [53].

$\operatorname{PPAR} \delta$, which has been shown to have increased activity in endothelial cells after resveratrol consumption in dosages of $20 \mathrm{mg} / \mathrm{kg} /$ day over a two-week span, contributes to the beneficial effect of SIRT1 to ameliorate endothelial dysfunction in diabetic and obese mice [56].

Additionally, PPAR $\delta$ activation by synthetic ligands protects against obesity, decreases triglyceride levels, increases HDL-cholesterol, and improves insulin sensitivity and could therefore also be helpful in regard to weight loss $[57,58]$. $150 \mathrm{mg} /$ day of resveratrol intake in 11 obese individuals over 30 days in a randomized double-blind crossover study resulted in metabolic changes mimicking the effects of calorie restriction [59]. These changes included reduced systolic blood pressure, adipose tissue lipolysis, plasma fatty acid, and glycerol levels and increased expression of AMPK, SIRT1, and PGC- $1 \alpha$ protein levels.

Increased expression of AMPK $\alpha$ by resveratrol treatment has also been found to be effective in elderly mice. In a study where 12 healthy, elderly mice were supplemented with $2.3 \mu \mathrm{g} / \mathrm{kg} /$ day of resveratrol for two consecutive days, fatty acid mobilization and degradation increased, and their synthesis was inhibited [22].

From this, we can conclude that resveratrol consumption can aid in weight loss and aging, prevent metabolic diseases before they arise, and improve endothelial metabolism in those with inflammatory states and cardiovascular-related diseases.

\section{Urolithin}

Urolithins are the major metabolites of dietary ellagic acid, found in the form of ellagitannin in foods such as walnuts, strawberries, and pomegranate. The three most abundant bioavailable ellagitannin metabolites are urolithins $\mathrm{A}, \mathrm{B}$, and $\mathrm{C}$, which are transformed by the gut bacteria Gordonibacter urolithinfaciens and Gordonibacter pamelaeae [60].

Numerous studies indicate that apart from their antiinflammatory effects, urolithin exhibits antiatherosclerotic and anticarcinogenic effects, which support their potential preventive effect against cardiovascular diseases $[61,62]$. One of the mechanisms by which quercetin acts is through inhibiting iNOS production and decreasing the 
expression of the proinflammatory cytokines IL- $1 \beta$, TNF- $\alpha$, and IL-6 $[63,64]$.

A double-blind randomized controlled crossover human intervention trial with 10 healthy males confirmed that ellagitannins acutely improve endothelial function through increasing flow-mediated dilation after the consumption of red raspberries in 200 and $400 \mathrm{~g}$ quantities when compared to a placebo group [65]. In another study where human artery endothelial cells were incubated with $50 \mu \mathrm{g} / \mathrm{mL}$ ox$\mathrm{LDL}$, urolithin A improved the productions of $\mathrm{NO}$ and eNOS in a dose-dependent manner [66]. Additionally, it significantly suppressed expressions of TNF- $\alpha$, IL-6, ICAM-1, MCP-1, and ET-1 and increased PPAR- $\gamma$ mRNA levels [66]. The beneficial effects attributed to the consumption of ellagitannin-containing foods against cardiovascular diseases were confirmed in another study where human aortic endothelial cells were exposed to TNF- $\alpha$ and treatment with Uro-A glucuronide inhibited monocyte adhesion and endothelial cell migration and decreased the expression of CCL-2 and IL-8 [61].

In vivo animal studies have shown that treatment with ellagic acid extracted from raspberry seed flour in concentrations estimated to $0.03 \%$ of the total diet lowers the elevated triglyceride and LDL cholesterol levels and oxidative stress provoked by a high-fat high-sucrose diet [67]. Another study showed that treatment of 7.5 and $15 \mathrm{mg} / \mathrm{kg}$ for 10 days reduced LDL, free fatty acids, triglyceride levels, and lipid peroxidation, while exerting cardiac protective effects by reducing ventricular hypertrophy and arrhythmia in myocardial infarction-induced rats [68].

In regard to obesity, urolithin $\mathrm{A}$ has been shown to reduce triglyceride accumulation, lipogenesis and gene expression related to adipocyte formation such as adiponectin, GLUT4, and FABP4 in 3T3-L1 adipocytes [69].

These results suggest that urolithin A has protective benefits against inflammation states and metabolic-related diseases, such as diabetes and obesity, which in turn are risk factors for cardiovascular disease and endothelial dysfunction.

The following table presents the mechanisms through which each of the four outlined polyphenols exert their protective effects as found through studies on humans, animals, and in vitro (Table 1).

\section{Discussion}

The exerted effects of catechin, quercetin, urolithin, and resveratrol on endothelial function are beneficial not only for patients with cardiovascular disease, diabetes, or endothelial dysfunction but also for all healthy individuals as it can improve the effects of aging on the endothelia and aid in weight loss, thereby further improving health status while lowering the risk of several diseases, including those caused by endothelial dysfunction.

In 2016, an article was published outlining the relation between a lower incidence of cardiovascular disease and the ingestion of antioxidants performed on 800 individuals in Holland. This effect was attributed to the consumption of flavonoids present in the diet, such as in apples, onion, and tea.
Although the exact compounds responsible for this effect were not determined, the authors described quercetin as one of them, as it was the compound with the highest antioxidant effect, due to its molecular structure [23]. Previous in vitro studies performed with human endothelial cells show that quercetin exerts not only antioxidant effects but also anti-inflammatory and vasodilator effects though downregulation of ACE [24]. Additionally, in these same cells, the antiinflammatory effects of quercetin mediated by a decrease in I-CAM, NF- $\kappa$ B, and AP-1 have been discovered [31, 32]. Furthermore, studies in vitro performed on rat liver cells have shown that quercetin diminishes the synthesis of fatty acids and triglycerides [26]. These data could explain the decrease in cardiovascular disease risk in the studies previously cited. However, the concentrations used in the studies in vitro are of micromolar magnitudes, which cannot be reached through diet and can only be achieved through supplementations. Toxicity studies have been performed in humans, where it has been stated that concentrations up to $1 \mathrm{~g} /$ day of quercetin does not pose any harm. Should one surpass this concentration however, quercetin could become prooxidant and thereby exert genotoxic effects [70].

The studies on the benefits of catechin also showed enhancements of the cardiovascular system through antioxidant, anti-inflammatory, antiproliferative, antithrombogenic, and antihyperlipidemic mechanisms [38]. The studies performed on humans with catechin and epigallocatechin supplements vary in dosages between 20 and $300 \mathrm{mg} / \mathrm{day}$, with variations in duration between 2 and 6 weeks, where increased flow-mediated dilation was found. These findings, along with those previously described, confirm that the consumption of catechin favors cardiovascular health [39]. Additionally, dietary intake of catechin through tea, grapes, or cacao has been proven to favor the increase in flow-mediated dilation in concentrations estimated to $146 \mathrm{mg}$ from cacao and $7.5 \mathrm{mg}$ from grapes. However, as these aliments contain a multitude of polyphenols, the suggested mechanisms on the cardiovascular system could be affected by other compounds. It is important to note that the outlined benefits were seen both in patients with cardiovascular diseases and obese individuals, as well as in young and elderly individuals $[39-41,44]$. Concerning dosage, there have been no reported findings of toxicity from catechin, although it is possible that it could follow the same guidelines as quercetin.

Resveratrol is one of the most studied polyphenols. It can be absorbed quickly due to its lipophilic characteristics and even more so when ingested with foods rich in fats or with alcohol such as red wine. However, it is quickly metabolized in the liver and therefore, its distribution is highly variable [71]. As shown in this review, resveratrol clearly improves lipidic profiles and endothelial smooth muscle relaxations in hypertense and obese patients $[54,55]$. In animal studies, it has been shown how this polyphenol increases lipid catabolism in skeletal muscle, which could possibly explain the changes in lipidic profiles in humans [71]. Moreover, studies have shown the protective effects of resveratrol in ischemic reperfusion-induced rats, mediated by autophagia and the mTOR signaling pathway [51]. In endothelial cells, resveratrol has been shown to decrease TNF- $\alpha$ levels and thereby 
TABLE 1: Outlined effects of quercetin, catechin, resveratrol, and urolithin studied in vitro, in animals, and in humans.

\begin{tabular}{|c|c|c|c|}
\hline Molecule & Study type & Mechanisms & Refs. \\
\hline \multirow{3}{*}{ Quercetin } & In vitro & $\begin{array}{c}\text { Reduces TNF- } \alpha \text {-induced expression of ICAM-1 } \\
\text { Inhibits VCAM- } 1 \text { and E-selectin expression, as well as } \\
\text { COX- } 2 \text { and iNOS } \\
\text { Activates the expression of HIF- } 1 \alpha \text { and regulates glucose and } \\
\text { hypoxia homeostasis }\end{array}$ & $\begin{array}{l}{[31]} \\
{[32]} \\
{[33]}\end{array}$ \\
\hline & Animal & $\begin{array}{l}\text { Induces reduction of fatty acid and triacylglycerol synthesis and } \\
\text { inhibits LDL oxidation } \\
\text { Increases Akt phosphorylation, eNOS phosphorylation, } \\
\text { and NO production } \\
\text { Increases expression of VEGF } \\
\text { Downregulates cytochrome p450 2b genes, steroid, } \\
\text { and cholesterol synthesis }\end{array}$ & $\begin{array}{c}{[26,27]} \\
{[28,29]} \\
{[30]} \\
{[34,35]}\end{array}$ \\
\hline & Human & Reduces cellular senescence & {$[25]$} \\
\hline \multirow{3}{*}{ Catechin } & In vitro & $\begin{array}{c}\text { Improves redox imbalance and mitochondrial dysfunction } \\
\text { by AMPK and ACC phosphorylation } \\
\text { Inhibits TNF- } \alpha \text {-induced insulin signaling pathway and induces } \\
\text { PGC-1 } \alpha \text { expression }\end{array}$ & $\begin{array}{l}{[47]} \\
{[48]}\end{array}$ \\
\hline & Animal & $\begin{array}{l}\text { Exerts beneficial effects on aortic expression of endothelial dysfunction } \\
\text { biomarkers (VCAM-1, ICAM-1, E-selectin, LOX-1, TNF- } \alpha \text {, and IL-6) }\end{array}$ & {$[46]$} \\
\hline & Human & $\begin{array}{l}\text { Increases flow-mediated dilation, reduces pulse wave velocity and } \\
\text { diastolic blood pressure } \\
\text { Increases thermogenesis, reduces adipogenesis, and promotes } \\
\text { fat oxidation }\end{array}$ & $\begin{array}{l}{[39-41]} \\
{[42,43]}\end{array}$ \\
\hline \multirow{3}{*}{ Resveratrol } & In vitro & $\begin{array}{l}\text { Reduces cholesterol peroxidation, reactive oxygen species levels, and platelet } \\
\text { aggregation by promoting vasodilation through NO synthesis and inhibition } \\
\text { of endothelin-1 } \\
\begin{array}{c}\text { Lowers proinflammatory mediators IL- } 8 \text { and ICAM- } 1 \text { in TNF- } \alpha \text {-activated } \\
\text { endothelial cells }\end{array}\end{array}$ & $\begin{array}{l}{[50-52]} \\
{[53]}\end{array}$ \\
\hline & Animal & $\begin{array}{l}\text { Ameliorates endothelial dysfunction through } \operatorname{PPAR} \delta \text { and } \\
\text { SIRT1 activation } \\
\text { Increases expression of AMPK } \alpha \text {, activates fatty acid mobilization and degradation, } \\
\text { and inhibits fatty acid synthesis }\end{array}$ & $\begin{array}{l}{[56]} \\
{[22]}\end{array}$ \\
\hline & Human & $\begin{array}{l}\text { Increases branchial flow-mediated dilation response } \\
\text { Rises the expression of AMPK, SIRT1, and PGC- } 1 \alpha \text { protein levels }\end{array}$ & $\begin{array}{l}{[54,55]} \\
{[59]}\end{array}$ \\
\hline \multirow[t]{3}{*}{ Urolithin } & In vitro & $\begin{array}{l}\text { Inhibits monocyte adhesion, endothelial cell migration, and CCL- } 2 \text { and } \\
\text { IL- } 8 \text { expressions } \\
\text { Inhibits iNOS and decreases proinflammatory cytokines expression } \\
\text { (IL- } 1 \beta \text {, TNF- } \alpha \text {, and IL-6) } \\
\text { Improves NO production and eNOS activity in a dose-dependent manner and } \\
\text { suppresses the expression of TNF- } \alpha \text {, IL-6, ICAM- } 1 \text {, MCP- } 1 \text {, and endothelin } 1 \\
\text { and increases PPAR- } \gamma \text { mRNA levels }\end{array}$ & $\begin{array}{c}{[61]} \\
{[63,64]} \\
{[66]}\end{array}$ \\
\hline & Animal & $\begin{array}{c}\text { Reduces LDL cholesterol, triglycerides, free fatty acids, and oxidative stress } \\
\text { Reduces ventricular hypertrophy and arrhythmia }\end{array}$ & $\begin{array}{l}{[67,68]} \\
{[68]}\end{array}$ \\
\hline & Human & Improves endothelial function through improvements in flow-mediated dilation & [65] \\
\hline
\end{tabular}

prevent inflammation [53], as well as protect against lipidic peroxidation in human monocytes [52]. Based on these findings, we can conclude that the authors of these studies agree that resveratrol exerts beneficial effects on the cardiovascular system.

Human studies relating urolithin to endothelial function are scarce. However, in one study performed on healthy individuals where $200-400 \mathrm{~g}$ of raspberry drink was administered, the presence of ellagitannins, the precursors of urolithin, was found present in the blood, in nanomolar magnitudes, with an augmented capacity for flow-mediated dilation [65], results similar to those found in quercetin. It is important to note that the exerted effects were likely not exclusively caused by urolithin, as the drink was rich in polyphenolic compounds. Interestingly, in the study performed on myocardial infarction-induced rats, although urolithin was found to exert protective benefits on the cardiovascular system, there was no significant difference between the groups administered 7.5 and $15 \mathrm{mg} /$ day, suggesting a saturation of the protective effects at the lower dosage. Moreover, in the control groups, there was no significant difference found in the lipidic profile in the nonsupplemented healthy animals and the healthy animals administered urolithin, suggesting that these dosages are not beneficial in healthy animals 


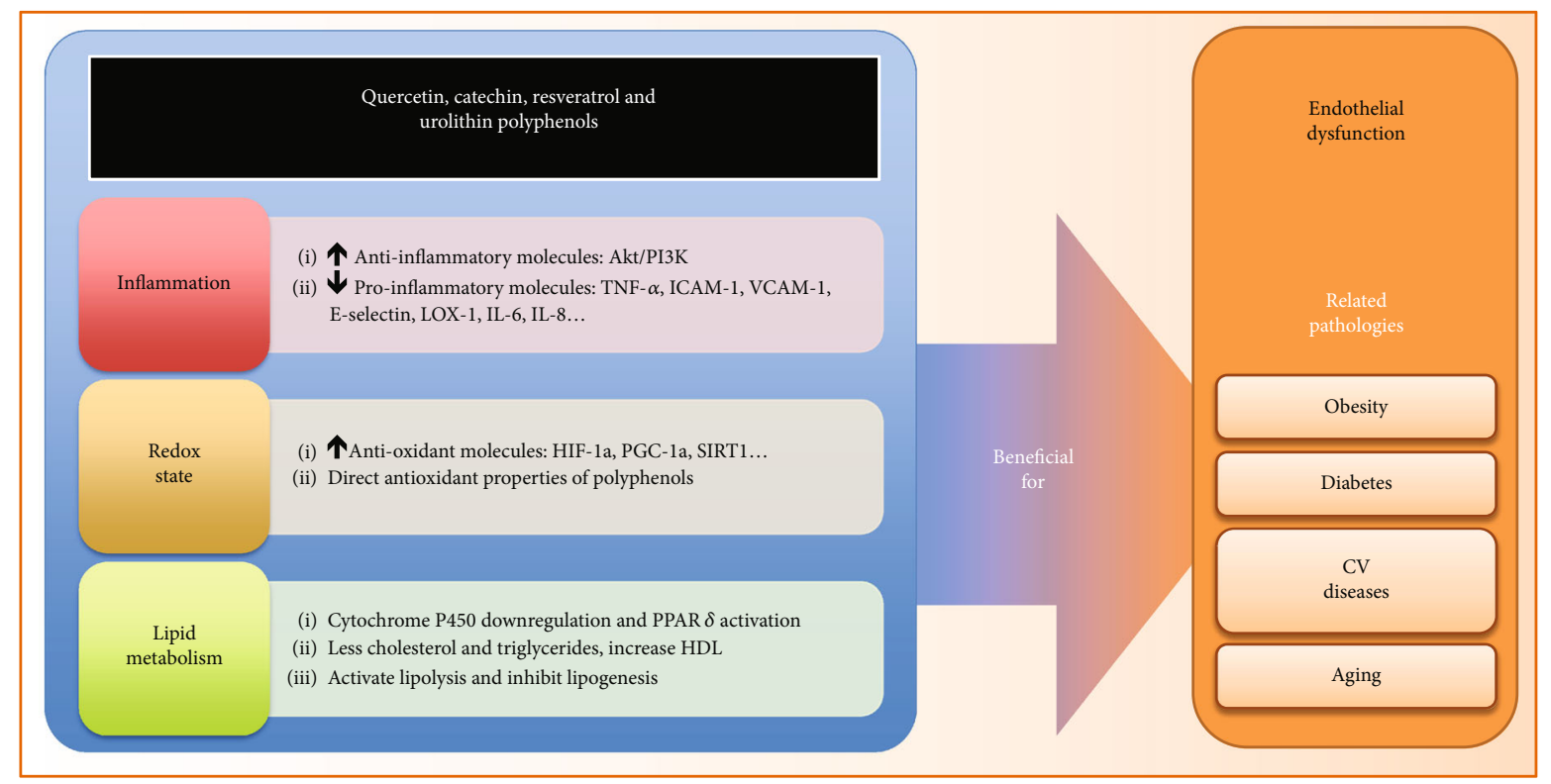

FIGURE 2: The effects of polyphenols in the Mediterranean diet on pathologies related to endothelial dysfunction.

[68]. Another study performed on animals administered a diet high in fats and sugar supplemented with $100 \mathrm{mg}$ of raspberry extract showed an improvement in lipidic profile and parameters of inflammation and oxidative stress [67]. It would be interesting to confirm these results with their concrete mechanisms and signaling pathways. The in vitro studies outlined in this review confirm some of these parameters. It has been shown how $5-15 \mu \mathrm{M}$ of urolithin exerts anti-inflammatory effects on endothelial cells [61]. Additionally, with these same concentrations, an anti-inflammatory pathway through activation of macrophages with lipopolysaccharides has been confirmed $[63,64]$. Other action mechanisms suggest a signaling pathway through microRNA-27 and ERK/PPAR- $\gamma$ in endothelial dysfunction caused by oxidized cholesterol treated with $5 \mu \mathrm{M}$ [66]. It is important to note that the concentrations of urolithin in the previously cited human study are administered in nanomolar magnitudes and it could therefore be interesting to perform studies in vitro to verify if the effects of these nutritionally relevant concentrations are beneficial.

The conditions in which polyphenols are consumed should also be taken into consideration; as for both quercetin and resveratrol, marked differences in functional effects were observed dependent on standard or high-fat dietary backgrounds [72]. As mentioned, it is important to note that the majority of the studies mentioned in this review were performed with polyphenols in supplemental quantities, rather than from natural plant sources, making the dosage recommendations for humans difficult to determine. Some of the amounts used in these trials may not be nutritionally relevant, and although no toxicity has been found in the articles outlined in this review when administered for up to 3 months, the extrapolation of the concentrations administered in some animal studies could prove to cause acute toxicity in humans. Further studies should be conducted with these polyphenols in quantities possible to achieve through diet. It would also be interesting to study these four polyphenols simultaneously, to see if they act upon common signaling pathways, if they have synergic effects, or if the beneficial effects could be increased when working together.

\section{Conclusion}

Thus, we can conclude that the polyphenols quercetin, catechin, resveratrol, and urolithin could be an effective tool, as they could serve as pharmacologic targets in treatments for diseases related to the cardiovascular system. These polyphenols can be used in combination with pharmacological treatments such as anti-inflammatory, anticoagulant, and antihypertensive drugs, to improve the damage caused by endothelial dysfunction (Figure 2). Additionally, they can exert protective effects in healthy individuals to reduce the risk of cardiovascular diseases.

\section{Abbreviations}

ACC: Acetyl-CoA carboxylase

AMPK: $\quad 5^{\prime}$ AMP-activated protein kinase

ANG II: Angiotensin II

CAMs: Cell adhesion molecules

ENOS: Endothelial nitric oxide synthase

EDHF: Endothelium-derived hyperpolarizing factor

EGF: Epidermal growth factor

E-selectin: Endothelial-leukocyte adhesion molecule 1

ET-1: Endothelin-1

FABP4: $\quad$ Fatty acid binding protein 4

FGF: $\quad$ Fibroblast growth factor

GLUT4: Glucose transporter type 4

H2S: $\quad$ Hydrogen sulfide

HSPG: Heparan sulfate proteoglycan

ICAM-1: Intercellular adhesion molecule-1

IIS: $\quad$ The insulin/IGF-1 signaling 
LDL: Low-density lipoprotein

LOX-1: Oxidized low-density lipoprotein receptor 1

MCP-1: $\quad$ Monocyte chemotactic protein 1

NO: $\quad$ Nitric oxide

PAI-1: Plasminogen activator inhibitor-1

PDGF: Platelet-derived growth factor

PGC-1 $\alpha$ : Peroxisome proliferator-activated receptor gamma coactivator 1-alpha

PGI2: $\quad$ Prostaglandin I2

PGH2: $\quad$ Prostaglandin $\mathrm{H} 2$

PPAR $\delta$ : Peroxisome proliferator-activated receptor gamma

ROS: $\quad$ Reactive oxygen species

SIIR: $\quad$ Steroid-induced insulin resistant

SIRT1: $\quad$ Sirtuin 1

TGF- $\beta$ : $\quad$ Transforming growth factor $\beta$

THP-1: Human acute monocytic leukemia cell line

TNF- $\alpha$ : Tumor necrosis factor- $\alpha$

tPA: $\quad$ Tissue plasminogen activator

TXA2: $\quad$ Thromboxane A2

VCAM-1: Vascular cell adhesion molecule 1

VEGF: Vascular endothelial growth factor

vWF: von Willebrand factor.

\section{Conflicts of Interest}

The authors declare no conflict of interest, financial, or otherwise.

\section{Authors' Contributions}

J.G. and K.S. have developed the concept of the manuscript, article conceptualization, and main contribution to introduction and conclusion sections. L.G.M., C.M.B., K.S., and J.G. have contributed to the bibliographic search, writing, and editing of different sections of the manuscript. K.S. and J.G. contributed to the abstract and in the review process. All authors reviewed the final manuscript.

\section{Acknowledgments}

This work was supported by FEDER (PIE15/00013), SAF201675508-R (Ministerio de 281 Economía y Competitividad), CB16/10/00435 (CIBERFES-ISCIII), PROMETEOII2014/056 282 (Conselleria de Educación, Investigación, Cultura y Deporte), the FRAILOMIC Initiative 283 (FP7-HEALTH2012-Proposal no. 305483-2), ADVANTAGE (724099) (HP-JA), DIALBFRAIL284 LATAM (825546 H2020-SC1BHC), and GV/2019/092 from "Conselleria d'Educació, Cultura i Esport de la Generalitat Valenciana" to C.M.-B. Finally, Dr. C.M.-B. is a recipient of a postdoctoral grant financed by Generalitat Valenciana (APOSTD/2018/230) and FSE (European Social Fund).

\section{References}

[1] P. Rajendran, T. Rengarajan, J. Thangavel et al., "The vascular endothelium and human diseases," International Journal of Biological Sciences, vol. 9, no. 10, pp. 1057-1069, 2013.

[2] S. Tribolo, F. Lodi, M. S. Winterbone et al., "Human metabolic transformation of quercetin blocks its capacity to decrease endothelial nitric oxide synthase (eNOS) expression and endothelin-1 secretion by human endothelial cells," Journal of Agricultural and Food Chemistry, vol. 61, no. 36, pp. 8589-8596, 2013.

[3] S. Moncada and A. Higgs, "The L-arginine-nitric oxide pathway," The New England Journal of Medicine, vol. 329, pp. 2002-2012, 1993.

[4] N. Suganya, E. Bhakkiyalakshmi, D. V. L. Sarada, and K. M. Ramkumar, "Reversibility of endothelial dysfunction in diabetes: role of polyphenols," The British Journal of Nutrition, vol. 116, no. 2, pp. 223-246, 2016.

[5] R. Corder, J. A. Douthwaite, D. M. Lees et al., "Endothelin-1 synthesis reduced by red wine," Nature, vol. 414, no. 6866, pp. 863-864, 2001.

[6] C. M. Sena, F. Carrilho, and R. M. Seiça, "Endothelial dysfunction in type 2 diabetes: targeting inflammation," in Endothelial Dysfunction - Old Concepts and New Challenges, 2018.

[7] A. Durazzo, M. Lucarini, E. B. Souto et al., "Polyphenols: a concise overview on the chemistry, occurrence, and human health," Phytotherapy Research, vol. 33, no. 9, pp. 2221-2243, 2019.

[8] D. M. Archivio, C. Filesi, R. Di Benedetto, R. Gargiulo, C. Giovannini, and R. Masella, "Polyphenols, dietary sources and bioavailability," Annali dell'Istituto Superiore di Sanità, vol. 43, no. 4, pp. 348-361, 2007.

[9] J. Dai and R. J. Mumper, "Plant phenolics: extraction, analysis and their antioxidant and anticancer properties," Molecules, vol. 15, no. 10, pp. 7313-7352, 2010.

[10] T. Ozdal, D. A. Sela, J. Xiao, D. Boyacioglu, F. Chen, and E. Capanoglu, "The reciprocal interactions between polyphenols and gut microbiota and effects on bioaccessibility," Nutrients, vol. 8, no. 2, p. 78, 2016.

[11] P. Ditano-Vázquez, J. D. Torres-Peña, F. Galeano-Valle et al., "The fluid aspect of the Mediterranean diet in the prevention and management of cardiovascular disease and diabetes: the role of polyphenol content in moderate consumption of wine and olive oil," Nutrients, vol. 11, no. 11, p. 2833, 2019.

[12] L. Schwingshackl, J. Morze, and G. Hoffmann, "Mediterranean diet and health status: active ingredients and pharmacological mechanisms," British Journal of Pharmacology, vol. 177, no. 6, pp. 1241-1257, 2020.

[13] M. Inglés, J. Gambini, M. G. Miguel et al., "PTEN mediates the antioxidant effect of resveratrol at nutritionally relevant concentrations," BioMed Research International, vol. 2014, 6 pages, 2014.

[14] C. López-Otín, M. A. Blasco, L. Partridge, M. Serrano, and G. Kroemer, "The hallmarks of aging," Cell, vol. 153, no. 6, pp. 1194-1217, 2013.

[15] J. C. Jeyapalan and J. M. Sedivy, "Cellular senescence and organismal aging," Mechanisms of Ageing and Development, vol. 129 , no. 7-8, pp. 467-474, 2008.

[16] G. Jia, A. R. Aroor, C. Jia, and J. R. Sowers, "Endothelial cell senescence in aging-related vascular dysfunction," Biochimica et Biophysica Acta - Molecular Basis of Disease, vol. 1865, no. 7, pp. 1802-1809, 2019.

[17] R. Bhayadia, B. M. W. Schmidt, A. Melk, and M. Hömme, "Senescence-induced oxidative stress causes endothelial dysfunction," The Journals of Gerontology: Series A, vol. 71, no. 2, pp. 161-169, 2016.

[18] S. A. Hawley, F. A. Ross, C. Chevtzoff et al., "Use of cells expressing $\gamma$ subunit variants to identify diverse mechanisms 
of AMPK activation," Cell Metabolism, vol. 11, no. 6, pp. 554$565,2010$.

[19] J. A. Baur, K. J. Pearson, N. L. Price et al., "Resveratrol improves health and survival of mice on a high-calorie diet," Nature, vol. 444, no. 7117, pp. 337-342, 2006.

[20] H. H. Ruiz, L. D. Raquel, A. Lakshmi, R. Ravichandran, and S. A. Marie, "Metabolism, obesity, and diabetes mellitus," Arteriosclerosis, Thrombosis, and Vascular Biology, vol. 39, pp. e166-e174, 2019.

[21] A. Engin, "Endothelial dysfunction in obesity," in Obesity and lipotoxicity. Advances in experimental medicine and biology, vol 960, Springer, Cham, 2017.

[22] L. Gimeno-Mallench, C. Mas-Bargues, M. Inglés et al., "Resveratrol shifts energy metabolism to increase lipid oxidation in healthy old mice," Biomedicine \& Pharmacotherapy, vol. 118, p. 109130, 2019.

[23] M. G. Hertog, E. J. Feskens, P. C. Hollman, M. B. Katan, and D. Kromhout, "Dietary antioxidant flavonoids and risk of coronary heart disease: the Zutphen Elderly Study," Lancet, vol. 342, no. 8878, pp. 1007-1011, 1993.

[24] W.-Y. Huang, L. Fu, C. Y. Li, L. P. Xu, L. X. Zhang, and W. M. Zhang, "Quercetin, hyperin, and chlorogenic acid improve endothelial function by antioxidant, antiinflammatory, and ACE inhibitory effects," Journal of Food Science, vol. 82, no. 5, pp. 1239-1246, 2017.

[25] L. J. Hickson, L. G. P. Langhi Prata, S. A. Bobart et al., "Senolytics decrease senescent cells in humans: preliminary report from a clinical trial of Dasatinib plus Quercetin in individuals with diabetic kidney disease," eBioMedicine, vol. 47, pp. 446-456, 2019.

[26] G. V. Gnoni, G. Paglialonga, and L. Siculella, "Quercetin inhibits fatty acid and triacylglycerol synthesis in rat-liver cells," European Journal of Clinical Investigation, vol. 39, no. 9, pp. 761-768, 2009.

[27] R. V. Patel, B. M. Mistry, S. K. Shinde, R. Syed, V. Singh, and H. S. Shin, "Therapeutic potential of quercetin as a cardiovascular agent," European Journal of Medicinal Chemistry, vol. 155, pp. 889-904, 2018.

[28] G. Zeng, F. H. Nystrom, L. V. Ravichandran et al., "Roles for insulin receptor, PI3-kinase, and Akt in insulin-signaling pathways related to production of nitric oxide in human vascular endothelial cells," Circulation, vol. 101, no. 13, pp. 1539-1545, 2000.

[29] X.-D. Guo, D. Y. Zhang, X. J. Gao et al., "Quercetin and quercetin-3-O-glucuronide are equally effective in ameliorating endothelial insulin resistance through inhibition of reactive oxygen species-associated inflammation," Molecular Nutrition \& Food Research, vol. 57, no. 6, pp. 1037-1045, 2013.

[30] N. Suganya, S. Dornadula, S. Chatterjee, and R. K. Mohanram, "Quercetin improves endothelial function in diabetic rats through inhibition of endoplasmic reticulum stress-mediated oxidative stress," European Journal of Pharmacology, vol. 819, pp. 80-88, 2018.

[31] H. Kobuchi, S. Roy, C. K. Sen, H. G. Nguyen, and L. Packer, "Quercetin inhibits inducible ICAM-1 expression in human endothelial cells through the JNK pathway," American Journal of Physiology-Cell Physiology, vol. 277, no. 3, pp. C403-C411, 1999.

[32] I. Crespo, M. V. García-Mediavilla, B. Gutiérrez, S. SánchezCampos, M. J. Tuñón, and J. González-Gallego, "A comparison of the effects of kaempferol and quercetin on cytokine-induced pro-inflammatory status of cultured human endothelial cells," The British Journal of Nutrition, vol. 100, no. 5, pp. 968-976, 2008.

[33] W. J. Wilson and L. Poellinger, "The dietary flavonoid quercetin modulates HIF- $1 \alpha$ activity in endothelial cells," Biochemical and Biophysical Research Communications, vol. 293, no. 1, pp. 446-450, 2002.

[34] E. F. Hoek-van den Hil, E. M. van Schothorst, I. van der Stelt et al., "Quercetin decreases high-fat diet induced body weight gain and accumulation of hepatic and circulating lipids in mice," Genes \& Nutrition, vol. 9, no. 5, p. 418, 2014.

[35] P. Manikandan and S. Nagini, "Cytochrome P 450 structure function and clinical significance: a review," Current Drug Targets, vol. 19, no. 1, pp. 38-54, 2018.

[36] H. V. Hwang, D. T. Tran, M. N. Rebuffatti, C.-S. Li, and A. A. Knowlton, "Investigation of quercetin and hyperoside as senolytics in adult human endothelial cells," PLoS One, vol. 13, no. 1, article e0190374, 2018.

[37] C. F. Haskell-Ramsay, J. Schmitt, and L. Actis-Goretta, "The impact of epicatechin on human cognition: the role of cerebral blood flow," Nutrients, vol. 10, no. 8, p. 986, 2018.

[38] A. A. Zanwar, S. L. Badole, P. S. Shende, M. V. Hegde, and S. L. Bodhankar, "Antioxidant role of catechin in health and disease," in Polyphenols in Human Health and Disease, 2014.

[39] R. Shafabakhsh, A. Milajerdi, Ž. Reiner et al., "The effects of catechin on endothelial function: a systematic review and meta-analysis of randomized controlled trials," Critical Reviews in Food Science and Nutrition, pp. 1-10, 2019.

[40] C. Heiss, R. Sansone, H. Karimi et al., "Impact of cocoa flavanol intake on age-dependent vascular stiffness in healthy men: a randomized, controlled, double-masked trial," Age, vol. 37, no. 3, p. 9794, 2015.

[41] J. Lekakis, L. S. Rallidis, I. Andreadou et al., "Polyphenolic compounds from red grapes acutely improve endothelial function in patients with coronary heart disease," European Journal of Cardiovascular Prevention \& Rehabilitation, vol. 12, no. 6, pp. 596-600, 2005.

[42] R. Hursel, W. Viechtbauer, A. G. Dulloo et al., "The effects of catechin rich teas and caffeine on energy expenditure and fat oxidation: a meta-analysis," Obesity Reviews, vol. 12, no. 7 , pp. e573-e581, 2011.

[43] Q. Shixian, B. VanCrey, J. Shi, Y. Kakuda, and Y. Jiang, "Green tea extract thermogenesis-induced weight loss by epigallocatechin gallate inhibition of catechol-O-methyltransferase," Journal of medicinal food, vol. 9, no. 4, pp. 451-458, 2006.

[44] G. Li, Y. Zhang, L. Thabane et al., "Effect of green tea supplementation on blood pressure among overweight and obese adults: a systematic review and meta-analysis," Journal of Hypertension, vol. 33, no. 2, pp. 243-254, 2015.

[45] Y. Mi, X. Liu, H. Tian et al., "EGCG stimulates the recruitment of brite adipocytes, suppresses adipogenesis and counteracts TNF- $\alpha$-triggered insulin resistance in adipocytes," Food \& Function, vol. 9, no. 6, pp. 3374-3386, 2018.

[46] C. Noll, J. Lameth, J.-L. Paul, and N. Janel, "Effect of catechi$\mathrm{n} /$ epicatechin dietary intake on endothelial dysfunction biomarkers and proinflammatory cytokines in aorta of hyperhomocysteinemic mice," European journal of nutrition, vol. 52, no. 3, pp. 1243-1250, 2013.

[47] A. Bartelt and J. Heeren, "Adipose tissue browning and metabolic health," Nature reviews Endocrinology, vol. 10, no. 1, pp. 24-36, 2014. 
[48] A. K. Doughan, D. G. Harrison, and S. I. Dikalov, "Molecular mechanisms of angiotensin II-mediated mitochondrial dysfunction," Circulation Research, vol. 102, no. 4, pp. 488-496, 2008.

[49] K. V. Kiselev, "Perspectives for production and application of resveratrol," Applied Microbiology and Biotechnology, vol. 90, no. 2, pp. 417-425, 2011.

[50] H. Ghanim, C. L. Sia, S. Abuaysheh et al., "An antiinflammatory and reactive oxygen species suppressive effects of an extract of Polygonum Cuspidatum containing resveratrol," The Journal of Clinical Endocrinology and Metabolism, vol. 95, no. 9, pp. E1-E8, 2010.

[51] N. Gurusamy, I. Lekli, S. Mukherjee et al., "Cardioprotection by resveratrol: a novel mechanism via autophagy involving the mTORC2 pathway," Cardiovascular Research, vol. 86, no. 1, pp. 103-112, 2010.

[52] H. Berrougui, G. Grenierad, S. Loued, G. Drouinad, and A. Khalil, "A new insight into resveratrol as an atheroprotective compound: inhibition of lipid peroxidation and enhancement of cholesterol efflux," Atherosclerosis, vol. 207, no. 2, pp. 420-427, 2009.

[53] I. M. Toaldo, J. Van Camp, G. B. Gonzales et al., "Resveratrol improves TNF- $\alpha$-induced endothelial dysfunction in a coculture model of a Caco-2 with an endothelial cell line," The Journal of nutritional biochemistry, vol. 36, pp. 21-30, 2016.

[54] B. C. Marques, M. Trindade, J. C. Aquino et al., "Beneficial effects of acute trans-resveratrol supplementation in treated hypertensive patients with endothelial dysfunction," Clinical and Experimental Hypertension, vol. 40, pp. 218-223, 2017.

[55] R. H. X. Wong, P. R. C. Howe, J. D. Buckley, A. M. Coates, I. Kunz, and N. M. Berry, "Acute resveratrol supplementation improves flow-mediated dilatation in overweight/obese individuals with mildly elevated blood pressure," Nutrition, Metabolism and Cardiovascular Diseases, vol. 21, no. 11, pp. 851-856, 2011.

[56] W. San Cheang, W. T. Wong, L. Wang et al., "Resveratrol ameliorates endothelial dysfunction in diabetic and obese mice through sirtuin 1 and peroxisome proliferator-activated receptor $\delta$," Pharmacological Research, vol. 139, pp. 384-394, 2019.

[57] T. Tanaka, J. Yamamoto, S. Iwasaki et al., "Activation of peroxisome proliferator-activated receptor induces fatty acid -oxidation in skeletal muscle and attenuates metabolic syndrome," Proceedings of the National Academy of Sciences, vol. 100, pp. 15924-15929, 2011.

[58] Y.-X. Wang, C. H. Lee, S. Tiep et al., "Peroxisome-proliferatoractivated receptor $\delta$ activates fat metabolism to prevent obesity," Cell, vol. 113, no. 2, pp. 159-170, 2003.

[59] S. Timmers, E. Konings, L. Bilet et al., "Calorie restriction-like effects of 30 days of resveratrol supplementation on energy metabolism and metabolic profile in obese humans," Cell Metabolism, vol. 14, no. 5, pp. 612-622, 2011.

[60] M. V. Selma, D. Beltrán, M. C. Luna et al., "Isolation of human intestinal bacteria capable of producing the bioactive metabolite isourolithin a from ellagic acid," Frontiers in Microbiology, vol. 8, 2017.

[61] J. A. Giménez-Bastida, A. González-Sarrías, M. Larrosa, F. Tomás-Barberán, J. C. Espín, and M.-T. García-Conesa, "Ellagitannin metabolites, urolithin A glucuronide and its aglycone urolithin A, ameliorate TNF- $\alpha$-induced inflammation and associated molecular markers in human aortic endothelial cells," Molecular nutrition \& food research, vol. 56, no. 5, pp. 784-796, 2012.
[62] V. Furlanetto, G. Zagotto, R. Pasquale, S. Moro, and B. Gatto, "Ellagic acid and polyhydroxylated urolithins are potent catalytic inhibitors of human topoisomerase II: an in vitro study," Journal of Agricultural and Food Chemistry, vol. 60, no. 36, pp. 9162-9170, 2012.

[63] J. P. Piwowarski, A. K. Kiss, S. Granica, and T. Moeslinger, "Urolithins, gut microbiota-derived metabolites of ellagitannins, inhibit LPS-induced inflammation in RAW 264.7 murine macrophages," Molecular Nutrition \& Food Research, vol. 59, no. 11, pp. 2168-2177, 2015.

[64] W. Komatsu, H. Kishi, K. Yagasaki, and S. Ohhira, "Urolithin $\mathrm{A}$ attenuates pro-inflammatory mediator production by suppressing PI3-K/Akt/NF- $\kappa$ B and JNK/AP-1 signaling pathways in lipopolysaccharide- stimulated RAW264 macrophages: possible involvement of NADPH oxidase-derived reactive oxygen species," European journal of pharmacology, vol. 833, pp. 411-424, 2018.

[65] G. Istas, R. P. Feliciano, T. Weber et al., "Plasma urolithin metabolites correlate with improvements in endothelial function after red raspberry consumption: a double-blind randomized controlled trial," Archives of Biochemistry and Biophysics, vol. 651, pp. 43-51, 2018.

[66] Q.-a. Han, C. Yan, L. Wang, G. Li, Y. Xu, and X. Xia, "Urolithin A attenuates ox-LDL-induced endothelial dysfunction partly by modulating microRNA-27 and ERK/PPAR- $\gamma$ pathway," Molecular Nutrition \& Food Research, vol. 60, no. 9, pp. 1933-1943, 2016.

[67] I. Kang, J. C. Espín, T. P. Carr, F. A. Tomás-Barberán, and S. Chung, "Raspberry seed flour attenuates high-sucrose dietmediated hepatic stress and adipose tissue inflammation," The Journal of Nutritional Biochemistry, vol. 32, pp. 64-72, 2016.

[68] M. M. Kannan and S. D. Quine, "Ellagic acid inhibits cardiac arrhythmias, hypertrophy and hyperlipidaemia during myocardial infarction in rats," Metabolism, vol. 62, no. 1, pp. 52-61, 2013.

[69] F. Les, J. M. Arbonés-Mainar, M. S. Valero, and V. López, "Pomegranate polyphenols and urolithin A inhibit $\alpha$-glucosidase, dipeptidyl peptidase-4, lipase, triglyceride accumulation and adipogenesis related genes in 3T3-L1 adipocyte-like cells," Journal of Ethnopharmacology, vol. 220, pp. 67-74, 2018.

[70] M. Harwood, B. Danielewska-Nikiel, J. F. Borzelleca, G. W. Flamm, G. M. Williams, and T. C. Lines, "A critical review of the data related to the safety of quercetin and lack of evidence of in vivo toxicity, including lack of genotoxic/carcinogenic properties," Food and Chemical Toxicology, vol. 45, no. 11, pp. 2179-2205, 2007.

[71] J. Gambini, M. Inglés, G. Olaso et al., "Properties of resveratrol: in vitro and in vivo studies about metabolism, bioavailability, and biological effects in animal models and humans," Oxidative Medicine and Cellular Longevity, vol. 2015, 13 pages, 2015.

[72] K. J. Pearson, J. A. Baur, K. N. Lewis et al., "Resveratrol delays age-related deterioration and mimics transcriptional aspects of dietary restriction without extending life span," Cell Metabolism, vol. 8, no. 2, pp. 157-168, 2008. 